\title{
ON THE ANGULAR LIMITS OF BLOCH FUNCTIONS
}

\author{
J.J. Carmona*, J. Curí and Ch. POMMERenKe**
}

Abstract

This paper contains a method to associate to each function $f$ in the little Bloch space another function $f^{*}$ in the Bloch space in such way that $f$ has a finite angular limit where $f^{*}$ is radially bounded. The idea of the method comes from the theory of the lacunary series. An application to conformal mapping from the unit disc to asymptotically Jordan domains is given.

\section{Introduction and main results}

Let $\mathrm{D}$ denote the unit disk and $\mathrm{T}=\partial \mathrm{D}$. A Bloch function $[1][8, \mathrm{p} .268]$ is a function $f$ analytic in $\mathrm{D}$ such that

$$
\|f\|_{B}=|f(0)|+\sup _{|z|<1}\left(1-|z|^{2}\right)\left|f^{\prime}(z)\right|<\infty .
$$

With this norm, the Bloch functions form a Banach space $B$. The closure in $B$ of the polynomials is a subspace $\mathcal{B}_{0}$ that consists of all $f \in B$ such that

$$
\left(1-|z|^{2}\right)\left|f^{\prime}(z)\right| \rightarrow 0 \text { as }|z| \rightarrow 1 .
$$

For Bloch functions, radial and angular limits are identical [7] [8, p. 268], that is,

$$
f(r \zeta) \rightarrow a(r \rightarrow 1) \Rightarrow f(z) \rightarrow a(z \rightarrow \zeta, z \in \Delta(\zeta))
$$

holds for each $\zeta \in \mathrm{T}$ where $\Delta(\zeta)$ is any triangle in $\mathrm{D}$ with vertex $\zeta$. Furthermore $[8$, p. 269]

$$
\sup _{0<r<1}|f(r \zeta)|<\infty \Rightarrow \sup _{z \in \Delta(\zeta)}|f(z)|<\infty
$$

-Supported in part by the grant PB85-0374 of the CICYT, Ministerio de Educacion y Ciencia, Spain.

- Supported in part by the Institut d'Estudis Catalans and by the Goethe-Institut. 
Each bounded analytic function belongs to $\mathcal{B}$ but not always to $\mathcal{B}_{0}$. Things are very simple for the special case of Hadamard gap series

$$
f(z)=\sum_{k=0}^{\infty} b_{k} z^{n_{k}}, \frac{n_{k+1}}{n_{k}} \geq \lambda>1 \quad(k=0,1, \ldots) .
$$

In this case $[1][13$, vol. I, p. 247]

$$
\begin{aligned}
& f \in \mathcal{B} \Longleftrightarrow \sup \left|b_{k}\right|<\infty, \\
& f \in \mathcal{B}_{0} \Longleftrightarrow b_{k} \rightarrow 0 \quad(k \rightarrow \infty), \\
& f \in H^{\infty} \Longleftrightarrow \sum_{k}\left|b_{k}\right|<\infty .
\end{aligned}
$$

If a gap series has radial limits on a set of positive measure then $\sum_{k}\left|b_{k}\right|^{2}<\infty$ $[13$, vol. I, p. 203]. It follows that

$$
f_{0}(z)=\sum_{k=1}^{\infty} k^{-1 / 2} z^{2^{k}} \quad(z \in \mathrm{D})
$$

belongs to $\mathcal{B}_{0}$ but has angular limits almost nowhere on $T$.

There is a close connection with conformal mappings [8, p. 269]. If $g$ is an (injective) conformal mapping of $\mathrm{D}$ then $f=c \log g^{\prime} \in \mathcal{B}$ holds for all $c \in \mathcal{C}$. Conversely if $f \in \mathcal{B}$ and $|c|<1 /\|f\|_{\mathcal{B}}$, then the function $g$ defined by $f=c \log g^{t}$ maps $D$ conformally onto a domain bounded by a Jordan curve $J$. Furthermore $f$ belongs to $\mathcal{B}_{0}$ if and only if [9] the curve $J$ is asymptotically conformal, i.e. if

$$
\max _{w \in J(a, b)} \frac{|b-w|+|w-a|}{|b-a|} \rightarrow 1 \text { as }|a-b| \rightarrow 0, a, b \in J
$$

where $J(a, b)$ is the (smaller) arc of $J$ between $a$ and $b$.

We shall describe a method to reduce the existence problem of finite radial (=angular) limits for $\mathcal{B}_{0}$ to the problem of radial boundedness for $\mathcal{B}$.

Theorem. If $f \in \mathcal{B}_{0}$ then there is a function $f^{*} \in \mathcal{B}_{0} \subset \mathcal{B}$ such that, for all $\zeta \in \mathbf{T}$,

$$
\sup _{r}\left|f^{*}(r \zeta)\right|<\infty \Rightarrow \lim _{r \rightarrow 1} f(r \zeta) \text { exists } \neq \infty .
$$

This generalizes a result on Hadamard gap series by Gnuschke [5]. We shall develop every Bloch function into a series of polynomials that is analogous to a gap series.

Using a method of Noshiro and T. Wolff [11], it can be shown [3] that each Bloch function is radially bounded on a set that has positive capacity on every arc of $\mathbf{T}$. Hence we obtain from the theorem: 
Corollary 1. If $f \in \mathcal{B}_{0}$ then there is a set $E \subset T$ with cap $(E \cap I)>0$ for every arc $I$ of $T$ such that

$$
\lim _{r \rightarrow 1} f(r \zeta) \text { exists } \neq \infty \text { for } \zeta \in E
$$

Previously it was only known [6] that a function in $\mathcal{B}_{0}$ has finite radial limits on an uncountably dense set. The present method, however, does not imply the fact [6] that the image set of angular limits has always positive linear measure.

It was asked in [4] whether all $f \in B$ satisfy

$$
\operatorname{dim}\left\{\zeta \in \mathrm{T}: \sup _{r}|f(r \zeta)|<\infty\right\}=1 \text {, }
$$

where dim denotes the Hausdorff dimension. If the answer turns out to be positive, then the theorem would imply that all $f \in \mathcal{B}_{0}$ have finite angular limits on a set of Hausdorff dimension 1 . This would be much stronger than our corollary because already $\operatorname{dim} E>0$ implies cap $E>0$. Note that it is not possible to replace dimension 1 by positive (Lebesgue) measure as the function $f_{0}$ defined by (4) shows.

Much more is known about infinite angular limits. Recently J.M. Anderson and L.D. Pitt [2] have proved that each Bloch function has either finite radial limits on a set of positive measure or satisfies

$$
\operatorname{dim}\{\zeta \in \mathrm{T}: \operatorname{Re} f(r \zeta) \rightarrow+\infty \text { as } r \rightarrow 1\}=1 .
$$

This implies that every conformal map has a finite angular derivative (possibly $=0$ ) on a set of dimension 1 .

Corollary 1 implies a result on the unrestricted boundary derivative for univalent functions; see [6] for the corresponding weaker resuit.

Corollary 2. Let g map $\mathbf{D}$ conformally onto the inner domain of an asymptotically conformal Jordan curve. Then there is a set $E \subset T$ with cap $(E \cap I)>0$ for every arc I of $\mathbf{T}$ such that

$$
g^{\prime}(\zeta)=\lim _{z \rightarrow \zeta, z \in \mathbf{D}} \frac{g(z)-g(\zeta)}{z-\zeta} \text { exists } \neq 0, \infty \text { for } \zeta \in E
$$

\section{A series expansion of Bloch functions}

We consider an analytic function

$$
f(z)=\sum_{n=0}^{\infty} a_{n} z^{n} \text { for } z \in \mathbf{D}
$$


and define polynomials $p_{0}(z)=a_{0}+a_{1} z+a_{2} z^{2}$ and

$$
p_{k}(z)=\sum_{n=2^{k-1}+2}^{2^{k}} \frac{2 n-2^{k}-2}{n-1} a_{n} z^{n}+\sum_{n=2^{k}+1}^{2^{k+2}} \frac{2^{k+1}-n+1}{n-1} a_{n} z^{n}
$$

for $k=1,2, \ldots$ Induction shows that

$$
\sum_{k=0}^{m} p_{k}(z)=\sum_{n=0}^{2^{m}} a_{n} z^{n}+\sum_{n=2^{m}+1}^{2^{m+1}} \frac{2^{m+1}-n+1}{n-1} a_{n} z^{n}
$$

and since lim sup $|a|^{1 / n} \leq 1$ it follows that

$$
f(z)=\sum_{k=0}^{\infty} p_{k}(z) \text { for } z \in \mathrm{D} .
$$

This expansion shares many properties of lacunary power series; see for instance (16) and Proposition 3 below.

The next two results are essentially known. They are implicit in the work of Zygmund [12] $[13$, vol. 1, p. $115 \mathrm{ff}]$ and actually hold in a slightly different form in the more general context of Besov spaces. For convenience we shall give proofs.

Proposition 1. If $f \in \mathcal{B}$ then

$$
\left\|p_{k}\right\|_{\infty} \equiv \sup _{|z| \leq 1}\left|p_{k}(z)\right| \leq 6\|f\|_{\mathfrak{B}} \text { for } k=0,1, \ldots,
$$

and if $f \in \mathcal{B}_{0}$ then

$$
\left\|p_{k}\right\|_{\infty} \rightarrow 0 \text { as } k \rightarrow \infty \text {. }
$$

Proof: We may assume that $\|f\|_{\mathcal{B}}=1$. It easily follows from (1) that $\left|a_{0}\right| \leq$ $1,\left|a_{1}\right| \leq 1$ and $\left|a_{2}\right|<2$ so that $\left|p_{0}(\zeta)\right| \leq 4$ for $|\zeta| \leq 1$. For $m=1,2, \ldots$, we consider now the polynomial

$$
q_{m}(z)=\left(\frac{1-z^{m}}{1-z}\right)^{2}=\sum_{\nu=0}^{m-1}(\nu+1) z^{\nu}+\sum_{\nu=m}^{2 m-2}(2 m-\nu-1) z^{\nu} .
$$

We see from (6) that

$$
\begin{aligned}
\frac{1}{2 \pi i} \int_{|z|=r} f^{\prime}(z) q_{m}(\bar{z} \zeta) z^{-2} d z & =\sum_{\nu=0}^{m-1}(\nu+1)(\nu+2) a_{\nu+2} r^{2 \nu} \zeta^{\nu}+ \\
& +\sum_{\nu=m}^{2 m-2}(2 m-\nu-1)(\nu+2) a_{\nu+2} r^{2 \nu} \zeta^{\nu} .
\end{aligned}
$$


A simple calculation therefore shows that

$$
\begin{aligned}
& \frac{1}{\pi} \iint_{\mathbf{D}}\left(1-|z|^{2}\right) f^{\prime}(z) q_{m}(\zeta \bar{z}) z^{-2} d x d y= \\
& =\sum_{n=2}^{m+1} a_{n} \zeta^{n-2}+\sum_{n=m+2}^{2 m} \frac{2 m-n+1}{n-1} a_{n} \zeta^{n-2} .
\end{aligned}
$$

Hence it follows from ( 7 ) that, for $k=1,2, \ldots$,

$$
p_{k}(\zeta)=\frac{1}{\pi} \iint_{D}\left(1-|z|^{2}\right) f^{\prime}(z)\left(\frac{\zeta}{z}\right)^{2}\left[q_{2^{k}}(\zeta \bar{z})-q_{2^{k-1}}(\zeta \bar{z})\right] d x d y .
$$

We write $y(r)=\max _{|z|=r}\left(1-|z|^{2}\right)\left|f^{\prime}(z)\right|$. Since

$$
\left|q_{2 m}(z)-q_{m}(z)\right|=\left|2 z^{m}+z^{2 m}\right|\left|\frac{1-z^{m}}{1-z}\right|^{2}
$$

by (11), we see from (12) that

$$
\begin{aligned}
\left|p_{k}(\zeta)\right| & \leq \frac{3}{\pi} \int_{0}^{1} y(r) r^{m-1}\left(\int_{0}^{2 \pi}\left|1+r e^{i t}+\cdots+r^{m-1} e^{i(m-1) t}\right|^{2} d t\right) d r \leq \\
& \leq 6 \int_{0}^{1} y(r) r^{m-1} m d r<6,
\end{aligned}
$$

with $m=2^{k-1}$, because $y(r) \leq 1$. If $f \in B_{0}$ and $\varepsilon>0$ then, by (2), there is $\rho<1$ such that $y(r)<\varepsilon$ for $\rho \leq r<1$. Hence the last integral is less thar $m \rho^{m+1}+\varepsilon<2 \varepsilon$ for large $m$ which implies (10).

Proposition 2. If $\left\|p_{k}\right\|_{\infty}$ is bounded then $f \in \mathcal{B}$ and

$$
\|f\|_{\mathcal{B}} \leq 16 \sup _{k \geq 0}\left\|p_{k}\right\|_{\infty}
$$

If $\left\|p_{k}\right\|_{\infty} \rightarrow 0$ as $k \rightarrow \infty$ then $f \in \mathcal{B}_{0}$.

Thus the Bloch norm is equivalent to the norm $\sup _{k}\left\|p_{k}\right\|_{\infty}$ where the polynomials $p_{k}$ are defined by (7). In the case of a lacunary series this norm is essentially the same as sup $\left|b_{k}\right|$.

Proof: Let $n_{k}=2^{k-1}$. If $k>0$ we can write

$$
p_{k}(z)=z^{n_{k}+1} g_{k}(z), \operatorname{deg}\left(g_{k}\right) \leq 3 n_{k}-1,\left\|g_{k}\right\|_{\infty}=\left\|p_{k}\right\|_{\infty} .
$$

Hence it follows from Bernstein's inequality $[13$, vol. II, p. 11] that, for $|z| \leq r<1$,

$$
\begin{aligned}
& \left|p_{k}^{\prime}(z)\right|=\left|\left(n_{k}+1\right) z^{n_{k}} g_{k}(z)+z^{n_{k}+1} g_{k}^{\prime}(z)\right| \leq \\
& \leq\left\{\left(n_{k}+1\right) r^{n_{k}}+\left(3 n_{k}-1\right) r^{n_{k}+1}\right]\left\|g_{k}\right\|_{\infty} \leq 4 n_{k} r^{n_{k}}\left\|p_{k}\right\|_{\infty} .
\end{aligned}
$$


Therefore we deduce from (1) and (8) that

$$
\|f\|_{\mathcal{B}} \leq 2\left\|p_{0}\right\|_{\infty}+\sup _{0 \leq r<1}\left(1-r^{2}\right) \sum_{k=1}^{\infty} 4 n_{k} r^{n_{k}}\left\|p_{k}\right\|_{\infty}
$$

Since (this is a standard estimate for gap series)

$$
\frac{1}{1-r} \sum_{k=1}^{\infty} n_{k} r^{n_{k}}=\sum_{m=1}^{\infty}\left(\sum_{n_{k} \leq m} n_{k}\right) r^{m} \leq 2 \sum_{m=1}^{\infty} m r^{m}=\frac{2 r}{(1-r)^{2}}
$$

we conclude that

$$
\|f\|_{B} \leq 2\left\|p_{0}\right\|_{\infty}+16 \sup _{k \geq 1}\left\|p_{k}\right\|_{\infty}
$$

which inplies 813 ). The final assertion of Proposition 2 is deduced in a similar way from (14).

Proposition 3. Let $f \in \mathcal{B}$. If $s_{k}=p_{0}+p_{1}+\cdots+p_{k}$ and $r_{k}=1-2^{-k}$ then

$$
\left|f\left(r_{k} z\right)-s_{k}(z)\right| \leq 30\|f\|_{\mathcal{B}} \text { for }|z| \leq 1 \text {. }
$$

Proof: We may assume that $\|f\|_{\mathcal{B}} \leq 1$. Then $\left\|p_{j}\right\|_{\infty} \leq 6$ by Proposition 1 . We see from (8) that, for $|z| \leq 1$,

$$
f\left(r_{k} z\right)-s_{k}(z)=\sum_{j=0}^{k}\left(p_{j}\left(r_{k} z\right)-p_{j}(z)\right)+\sum_{j=k+1}^{\infty} p_{j}\left(r_{k} z\right) .
$$

The first sum is bourded by

$$
\sum_{j=0}^{k}\left(1-r_{k}\right) \max _{|\zeta| \leq I}\left|p_{k}^{\prime}(\zeta)\right| \leq 2^{-k} \sum_{j=0}^{k} 4 \cdot 2^{j-1} \cdot 6<24
$$

because of (15), and we see from (14) that the second sum is bounded by

$$
6 \sum_{j=k+1}^{\infty} r_{k}^{2^{j-1}}<6 \sum_{j=k+1}^{\infty} \exp \left(-2^{j-1-k}\right)=6 \sum_{\nu=0}^{\infty} \exp \left(-2^{\nu}\right)<6
$$

\section{Proof of the main results}

Proof of the theorem: Let $f \in \mathcal{B}_{0}$. We obtain from Proposition 1 that there is a decreasing sequence $\left(\varepsilon_{k}\right)$ such that

$$
\left\|p_{k}\right\|_{\infty}<\varepsilon_{k}^{2} \text { for } k=0,1, \ldots
$$


where $p_{k}$ is given by (7). We define

$$
f^{*}=\sum_{k=0}^{\infty} p_{k}^{*}, \quad p_{k}^{*}=\varepsilon_{k}^{-1} p_{k} \quad(k=0,1, \ldots) .
$$

This coincides with the expansion (8) of $f^{*}$.

Since $\left\|p_{k}^{*}\right\|_{\infty}<\varepsilon_{k}$ by (18), we conclude from Proposition 2 that $f^{*} \in \mathcal{B}_{0}$.

Writing $s_{k}^{*}=p_{0}^{*}+p_{1}^{*}+\cdots+p_{k}^{*}$, a partial summation gives

$$
\sum_{k=0}^{N} \varepsilon_{k} p_{k}^{*}=\varepsilon_{N} s_{N}^{*}+\sum_{k=0}^{N-1}\left(\varepsilon_{k}-\varepsilon_{k+1}\right) s_{k}^{*} .
$$

Let now $\left|f^{*}(r \zeta)\right|$ be bounded in $0 \leq r<1$ for some $\zeta \in \mathbf{T}$. Proposition 3 implies that $\left|s_{k}^{*}(\zeta)\right|$ is aiso bounded in $k$. Since $s_{k}^{*}(r \zeta)$ is continuous in $0 \leq r<1$ for each $k$ and since $\varepsilon_{k}-\varepsilon_{k+1} \geq 0$, we easily deduce that

$$
f(r \zeta)=\sum_{k=0}^{\infty}\left(\varepsilon_{k}-\varepsilon_{k+1}\right) s_{k}^{*}(r \zeta)
$$

is uniformly continuous in $0 \leq r<1$ and therefore has a finite limit as $r-1$.

Proof of the Corollary: The function $f=\log g^{\prime}$ belongs to $B_{0}$ and therefore has a finite radial limit on a set $E \subset \mathrm{T}$ with cap $(E \cap I)>0$ for every arc $I$ of T.

Let now $\zeta \in E$. Then $g^{\prime}$ has a finite nonzero radial limit at $\zeta$ and it follows $[8$, p. 305$]$ that

$$
\lim _{r \rightarrow 1} \frac{g(r \zeta)-g(\zeta)}{(r-1) \zeta} \neq 0, \infty
$$

exists. Since $J$ is asymptotically conformal, we conclude from a theorem of Warschawski [10, Satz II] or from [9, Corollary 3$]$ that the unrestricted derivative exists.

\section{References}

1. J.M. Anderson, J. Clunie, Ch. Pommerenke, On Bloch functions and normal functions, $J$. reine angew. Math. 270 (1974), 12-37.

2. J.M. ANDERSON, L.D. PITT, The boundary behaviour of Bloch functions and univalent functions, preprint 1987.

3. J.J. CARMONA, J. Cuff, CH. POMMERENKe, On the angular boundedness of Bloch functions, preprint 1988.

4. J.L. FERNÁNDEZ, CH. POMMERENKE, On the boundary behavior of Bloch functions, Proceedings of Berkeley conference (1986), to appear. 
5. D. GNUSCHKE, On power series with Hadamard gaps, Analysis 4 (1984), 61-72.

6. D. Gnuschke-Hauschild, Ce. Pommerenke, On Bloch functions and gap series, $J$. reine angew. Math. 367 (1986), 172-186.

7. O. LEHTO, K.J. VIRTANEN, Boundary behaviour and normal meromorphic functions, Acta Math. 97 (1957), 47-65.

8. Ch. POMMERENKe, "Univalent functions," Vandenhoeck-Ruprecht, Göttingen, 1975.

9. Ch. POMMERENKe, On univalent functions, Bloch functions and $V M O A$, Math. Ann. 236 (1978), 199- 208.

10. S.E. WARSCHAWSKI, Zur Randverzerrung bei konformer Abbildung, Compos. Math. 1 (1935), 314-330.

11. T. WOLFF, written communication.

12. A. ZYGmund, Smooth functions, Duke Math. J. 12 (1945), 47-76.

13. A. ZYGMUND, "Trigonometric series," Cambridge University Press, 1968.

\author{
J.J. Carmona: Departament de Matemàtiques \\ Universitat Autònoma de Barcelona \\ 08193 Bellaterra, SPAIN. \\ J. Cufi: Departament de Matemàtiques \\ Universitat Autònoma de Barcelona. \\ 08193 Bellaterra, SPAIN. \\ Ch. Pommerenke: Fachbereich Mathematik \\ Technische Universität \\ D-1000 Berlin 12, WEST GERMANY.
}

Rebut el 12 de Marৎ de 1988 